\title{
Cognitive outcome in adults after bacterial meningitis
}

\author{
Martine Hoogman, Diederik van de Beek, Martijn Weisfelt, Jan de Gans, Ben Schmand
}

J Neurol Neurosurg Psychiatry 2007;78:1092-1096. doi: 10.1136/jnnp.2006.110023

See end of article for authors' affiliations

.....................

Correspondence to: Dr Diederik van de Beek, Department of Neurology, Academic Medical Centre

University of Amsterdam, PO

Box 22660, 1100 DD

Amsterdam, the

Netherlands; d.vandebeek@

amc.uva.nl

Received 27 October 2006

Revised 17 January 2007

Accepted 22 February 2007

Published Online First

12 March 2007

....................

\begin{abstract}
Objective: To evaluate cognitive outcome in adult survivors of bacterial meningitis.
Methods: Data from three prospective multicentre studies were pooled and reanalysed, involving 155 adults surviving bacterial meningitis (79 after pneumococcal and 76 after meningococcal meningitis) and 72 healthy controls.

Results: Cognitive impairment was found in $32 \%$ of patients and this proportion was similar for survivors of pneumococcal and meningococcal meningitis. Survivors of pneumococcal meningitis performed worse on memory tasks $(p<0.001)$ and tended to be cognitively slower than survivors of meningococcal meningitis $(p=0.08)$. We found a diffuse pattern of cognitive impairment in which cognitive speed played the most important role. Cognitive performance was not related to time since meningitis; however, there was a positive association between time since meningitis and self-reported physical impairment $(p<0.01)$. The frequency of cognitive impairment and the numbers of abnormal test results for patients with and without adjunctive dexamethasone were similar.

Conclusions: Adult survivors of bacterial meningitis are at risk of cognitive impairment, which consists mainly of cognitive slowness. The loss of cognitive speed is stable over time after bacterial meningitis; however, there is a significant improvement in subjective physical impairment in the years after bacterial meningitis. The use of dexamethasone was not associated with cognitive impairment.
\end{abstract}

$\mathrm{T}$ he estimated annual incidence of bacterial meningitis is 46 per 100000 adults and Streptococcus pneumoniae (pneumococcus) and Neisseria meningitidis (meningococcus) are the causative bacteria in $80 \%$ of cases. ${ }^{12}$ Fatality rates in patients with pneumococcal meningitis (26\%) and meningococcal meningitis $(7 \%)$ are significant. ${ }^{1-3}$ Even in patients with apparent good recovery, cognitive impairment occurs frequently, ${ }^{4}$ especially after pneumococcal meningitis. ${ }^{4-6}$ The cognitive functions affected by bacterial meningitis differ between studies, most likely because of the limited numbers of patients examined, and the lack of uniformity across studies in assessment methods and in the definition of cognitive impairment. ${ }^{4-10}$ We therefore pooled data on cognitive outcome after bacterial meningitis from three of our previous studies to more clearly determine which cognitive functions are affected by bacterial meningitis and to identify which patients are at risk of developing cognitive impairment.

\section{METHODS}

\section{Selection of patients}

Data on neuropsychological evaluations were derived from three long term follow-up studies embedded in two research projects: the European Dexamethasone Study (EDS) and the Dutch Meningitis Cohort. ${ }^{11}$ The total number of patients that underwent neuropsychological evaluation in these studies was 155 (79 patients after pneumococcal and 76 patients after meningococcal meningitis ). ${ }^{4}{ }^{10}$ A group of 72 healthy subjects formed the control group. The distribution of scores on the Glasgow Outcome Scale (GOS) was as follows: 129 patients had a GOS score of 5 (good recovery), 25 patients had a GOS score of 4 (moderate disability) and one patient had a GOS score of 3 (severe disability). Participants gave written informed consent and all studies were approved by the local ethics committee. ${ }^{1461011}$

The EDS was a randomised, placebo controlled, double blind trial of adjunctive dexamethasone therapy in adults with bacterial meningitis; 301 patients were included between June 1993 and December 2001. ${ }^{11}$ Part of this sample $(n=87$;
38 pneumococcal and 49 meningococcal patients) participated in a long term follow-up study on cognitive outcome. ${ }^{6}$ Patients eligible for this neuropsychological study were survivors of pneumococcal or meningococcal meningitis, confirmed by CSF culture, who were aged $>17$ years; exclusion criteria were other serious illness (interfering with cognitive testing), pre-existing psychiatric disorders, insufficient mastery of the Dutch language and evidence of alcohol or other substance abuse. Eighty-one patients had a GOS score of 5 (35 after pneumococcal and 46 after meningococcal meningitis), five patients had a GOS score of 4 (two after pneumococcal and three after meningococcal meningitis) and one pneumococcal patient had a GOS score of 3. The control subjects were partners, relatives or close friends of the patients $(n=50)$.

The Dutch Meningitis Cohort was a prospective observational cohort study of adults with community acquired bacterial meningitis; 696 episodes were included between October 1998 and April 2002. ${ }^{1}$ Two follow-up studies on neuropsychological outcome were conducted in some of this cohort. ${ }^{40}$ Patients were aged 16-65 years and exclusion criteria were as mentioned above. Forty-eight patients had a GOS score of 5 (21 after pneumococcal and 27 after meningococcal meningitis) and 20 patients had a GOS score of 4 (all after pneumococcal meningitis). The total number of participants was 68 patients (41 after pneumococcal and 27 after meningococcal meningitis). A group of control subjects consisted of partners, relatives or close friends $(n=25)$. Three controls were included in both studies. ${ }^{410}$

\section{Neuropsychological evaluation}

In each study, patients were tested with an identical battery of standardised neuropsychological tests, which has been described previously. ${ }^{4610}$ The battery included the following tests.

Abbreviations: EDS, European Dexamethasone Study; GIT, Groningen Intelligence Test; GOS, Glasgow Outcome Scale; POMS, Profile of Mood States 
Table 1 Clinical characteristics of patients with pneumococcal and meningococcal meningitis

\begin{tabular}{|c|c|c|c|}
\hline & $\begin{array}{l}\text { Pneumococcal } \\
\text { patients }(n=79)\end{array}$ & $\begin{array}{l}\text { Meningococcal } \\
\text { patients }(n=76)\end{array}$ & p Value* \\
\hline \multicolumn{4}{|l|}{ Presentation } \\
\hline Glasgow Coma Scale score (mean (SD)) & $11(3)$ & $12(3)$ & 0.03 \\
\hline Coma (n (\%)) & $10(13)$ & $8(11)$ & $0.62 t$ \\
\hline Median heart rate $(\mathrm{bpm})$ (mean (SD)) & $100(16.8)$ & $88(18.9)$ & $<0.001$ \\
\hline Diastolic blood pressure $<60 \mathrm{~mm} \mathrm{Hg}$ (n (\%)) & $8(10)$ & $16(21)$ & 0.12 \\
\hline Focal cerebral deficitsł (n (\%)) & $18(23)$ & 11 (14) & $0.22 t$ \\
\hline Cranial nerve palsies (n (\%)) & $16(20)$ & $5(7)$ & $0.02 t$ \\
\hline \multicolumn{4}{|l|}{ Laboratory results on admission } \\
\hline CSF leucocyte count $\left(\times 10^{3}\right.$ cells $/ \mathrm{mm}^{3}$ ) (mean (SD)) & $18(25)$ & $19(28)$ & 0.42 \\
\hline CSF protein level (g/l) (mean (SD)) & $4.3(2.7)$ & $4.9(3.4)$ & 0.43 \\
\hline CSF glucose level (mg/dl) (mean (SD)) & $1.2(1.4)$ & $1.4(1.9)$ & 0.62 \\
\hline Positive blood culture (n (\%)) & $54(68)$ & $56(74)$ & $0.99+$ \\
\hline \multicolumn{4}{|l|}{ Focal neurological abnormalities at discharge } \\
\hline Focal cerebral deficits (n (\%)) & $4(5)$ & $2(3)$ & $0.68+$ \\
\hline Cranial nerve palsies (n (\%)) & $22(28)$ & $9(12)$ & $0.02 \dagger$ \\
\hline Glasgow Outcome Scale score 3 or $4 / 5$ at discharge & $23 / 56$ & $3 / 73$ & $<0.001 \dagger$ \\
\hline Time between illness and cognitive testing (months) (mean (SD)) & $54.7(44.0)$ & $68.8(49.4)$ & 0.10 \\
\hline
\end{tabular}

(1) Memory: Rey's Auditory Verbal Learning Test, ${ }^{12}$ Rivermead Behavioural Memory Test subtest story recall and Wechsler Memory Scale Revised subtest visual reproduction. ${ }^{13}$

(2) Attention/executive functions: colour-word card of the Stroop Test, ${ }^{14}$ numerical speed of the Groningen Intelligence Test (GIT), Trail Making Test part B, ${ }^{15}$ Category Fluency, Letter Fluency and Wisconsin Card Sorting Test. ${ }^{16}$

(3) Psychomotor functions: Trail Making Test part A, ${ }^{15}$ word and colour cards of the Stroop Test, simple and two choice reaction tasks.

(4) Intelligence: Abbreviated version of the $\mathrm{GIT}^{17}$ containing verbal and visuospatial reasoning. The Dutch Adult Reading Test was used as an estimator of premorbid intelligence. $^{18}$

(5) Questionnaires: The Profile of Mood States (POMS) ${ }^{19}$ was used to identify the presence of depressive mood disorders. Additionally, the RAND- $36^{2021}$ was administrated to evaluate quality of life and general health.

\section{Statistical analysis}

All test scores were expressed as T scores corrected for age and education with the control group as reference. Outliers of individual test scores of the control group were excluded $(<1 \%$ of raw neuropsychological data points). Skewed score distributions were normalised with appropriate transformations. Linear multiple regression analyses were conducted with the raw (or transformed) test score of the controls as the dependent variable, and age and educational level as independent variables. The resulting regression formulas were used to calculate expected test scores for each subject on each test. Differences between expected and observed scores were divided by the standard error of the estimate and transformed to $\mathrm{T}$ scores (mean 50 (SD 10); a higher score indicating better performance).

To examine relative differences between groups, multivariate analyses of variance (MANOVAs) were performed within each cognitive domain using $\mathrm{T}$ scores. If these MANOVAs revealed significant results (Pillais' trace two tailed $\mathrm{p}<0.05$ ), t tests with Bonferroni correction for the number of comparisons were conducted to evaluate single neuropsychological measures within each cognitive domain. Subsequently, we evaluated the cognitive profile of meningitis patients; an individual test score was defined as impaired if it was at least two SDs below the mean normative score of our control group ( $\mathrm{T}$ score $<30$ ). Cognitive impairment was considered to be present if performance, reflected by the number of impaired test results, was worse than the fifth percentile of our control group. To compare cognitive speed between groups, a speed composite score was calculated for each participant by taking the mean $\mathrm{T}$ score of tests in which cognitive speed is important, as described previously. ${ }^{10}$

For non-parametric testing, the Mann-Whitney $U, \chi^{2}$ or Fisher's exact statistics were used. Spearman correlations were calculated between cognitive performance and depression, GOS score, time since meningitis and physical functioning.

We performed a logistic regression analysis to identify determinants of cognitive impairment. Independent variables in this analysis were clinical and demographic characteristics and the dependent variable was presence of cognitive impairment.

\section{RESULTS}

\section{Clinical characteristics}

Patients with pneumococcal meningitis were admitted with a higher severity of disease, as reflected by lower scores on the

Table 2 Demographic characteristics of patients and controls

\begin{tabular}{lllll}
\hline & $\begin{array}{l}\text { Pneumococcal patients } \\
(\mathbf{n}=79)\end{array}$ & $\begin{array}{l}\text { Meningococcal patients } \\
(\mathbf{n}=76)\end{array}$ & $\begin{array}{l}\text { Controls } \\
(\mathbf{n}=72)\end{array}$ & p Value* \\
\hline Age at follow-up $(\mathrm{y})$ & $52.3(13.6)$ & $38.9(16.6)$ & $46.6(16.0)$ & $<0.01$ \\
Years of education & $12.4(2.4)$ & $13.3(1.8)$ & $13.1(1.5)$ & 0.01 \\
Premorbid IQ & $98.6(16.7)$ & $98.0(15.8)$ & $99.4(16.7)$ & 0.97 \\
Sex (M/F) & $40 / 39$ & $40 / 36$ & $21 / 50$ & $0.01 \dagger$ \\
\hline Values are mean (SD) or number. & & & \\
*Two tailed $p$ value of ANOVA; $+\chi^{2}$ test. & & & \\
\hline
\end{tabular}


Table 3 T scores of neuropsychological tests in survivors of bacterial meningitis

\begin{tabular}{|c|c|c|c|c|c|}
\hline \multirow[b]{2}{*}{ Neuropsychological measure } & \multirow{2}{*}{$\begin{array}{l}\text { Pneumococcal } \\
\text { patients }(n=79)\end{array}$} & \multirow{2}{*}{$\begin{array}{l}\text { Meningococcal } \\
\text { patients }(n=76)\end{array}$} & \multicolumn{3}{|c|}{ p Value* } \\
\hline & & & Pvs $M$ & P vs C & $M$ vs $C$ \\
\hline \multicolumn{6}{|l|}{ Intelligence } \\
\hline Visuospatial reasoning (GIT) & $50.5(10.9)$ & $50.0(11.4)$ & 0.77 & 0.78 & 0.97 \\
\hline Verbal reasoning (GIT) & $50.8(10.5)$ & $51.2(10.6)$ & 0.79 & 0.62 & 0.45 \\
\hline \multicolumn{6}{|l|}{ Aftention/executive functioning } \\
\hline Stroop Colour-Word Card & $44.1(13.8)$ & $45.9(11.1)$ & 0.39 & 0.01 & 0.04 \\
\hline TMT part B & $40.4(17.4)$ & $45.6(12.6)$ & 0.04 & 0.00 & 0.05 \\
\hline Numerical ability (GIT) & $46.8(10.4)$ & $51.8(10.5)$ & $<0.01$ & 0.06 & 0.31 \\
\hline Category Fluency & $50.9(10.8)$ & $48.9(12.6)$ & 0.30 & 0.63 & 0.55 \\
\hline Letter Fluency & $47.4(10.7)$ & $48.1(7.9)$ & 0.67 & 0.14 & 0.21 \\
\hline WCST categories & $48.0(13.4)$ & $46.4(11.0)$ & 0.42 & 0.40 & 0.07 \\
\hline WCST errors & $49.8(10.8)$ & $48.1(10.5)$ & 0.34 & 0.92 & 0.28 \\
\hline WCST perseverative errors & $46.9(12.2)$ & $46.3(10.2)$ & 0.74 & 0.11 & 0.03 \\
\hline \multicolumn{6}{|l|}{ Memory } \\
\hline RMBT immediate recall & $50.4(9.2)$ & $50.6(12.1)$ & 0.92 & 0.78 & 0.73 \\
\hline RMBT \% retained & $48.5(13.0)$ & 46.3 (13.3) & 0.33 & 0.30 & 0.04 \\
\hline WMS immediate recall & $47.0(14.2)$ & $48.6(11.8)$ & 0.48 & 0.16 & 0.46 \\
\hline WMS \% retained & 45.7 (15.9) & $51.5(9.3)$ & $<0.01$ & 0.06 & 0.36 \\
\hline AVLT immediate recall & $48.2(10.2)$ & $48.8(10.2)$ & 0.72 & 0.10 & 0.21 \\
\hline AVLT \% retained & $48.8(13.6)$ & $48.0(13.0)$ & 0.81 & 0.66 & 0.48 \\
\hline \multicolumn{6}{|l|}{ Psychomotor functions } \\
\hline TMT part A & $43.0(14.6)$ & $47.7(11.3)$ & 0.03 & 0.00 & 0.37 \\
\hline Stroop Word-Card & 43.9 (14.4) & $45.6(13.3)$ & 0.46 & 0.00 & 0.03 \\
\hline Stroop Colour-Card & $43.2(11.1)$ & $43.7(12.3)$ & 0.80 & 0.00 & 0.00 \\
\hline SRT dominant hand & $40.6(18.0)$ & 44.8 (13.3) & 0.12 & 0.00 & 0.02 \\
\hline SRT non-dominant hand & $43.3(17.2)$ & $45.9(11.2)$ & 0.31 & 0.02 & 0.05 \\
\hline Two choice reaction time & $47.2(17.2)$ & $48.4(12.1)$ & 0.65 & 0.31 & 0.44 \\
\hline Speed score & $45.3(8.8)$ & $47.5(6.7)$ & 0.08 & $<0.01$ & 0.06 \\
\hline No of impaired tests & $2.2(2.3)$ & $1.6(2.1)$ & 0.10 & $<0.01$ & $<0.01$ \\
\hline \multicolumn{6}{|c|}{$\begin{array}{l}\text { AVLT, Rey Auditory Verbal Learning Test; GIT, Groningen Intelligence Test; RBMT, Rivermead Behavioural Memory Test; } \\
\text { SRT, Simple Reaction Time; TMT, Trail Making Test; WCST, Wisconsin Card Sorting Test; WMS, Wechsler Memory } \\
\text { Scale. } \\
\text { Values are mean (SD). } \\
\text { *Two tailed p value of } t \text { test: comparison of pneumococcal (P) and meningococcal (M) patients; comparison of } \\
\text { pneumococcal (P) patients and controls (C); comparison of meningococcal (M) patients and controls (C). }\end{array}$} \\
\hline
\end{tabular}

Glasgow Coma Scale (table 1). Laboratory examination showed no significant differences between groups. Initial antimicrobial treatment consisted of penicillin or amoxicillin for $70 \%$ of episodes, third generation cephalosporins in $8 \%$, cephalosporins in combination with penicillin or amoxicillin in $17 \%$ of episodes and another regimen in 5\%; $30 \%$ received adjunctive dexamethasone treatment.

\section{Cognitive impairment}

Patients did not differ from controls with respect to age $(p=0.72)$, years of education $(p=0.66)$ or premorbid intelligence $(p=0.81)$ (table 2$)$.

We found significant differences between patients and controls. MANOVAs within each cognitive domain showed significant overall group differences between patients and controls for "attention/executive functions" (table 3; $p=0.03$ ) and for "psychomotor functions" $(p=0.03)$ but not for "intelligence" $(p=0.77)$ and "memory" $(p=0.21)$. Patients performed worse than controls on TMT part B $(\mathrm{p}<0.01)$, Colour-Word Card of the Stroop $(\mathrm{p}<0.01)$ and Simple Reaction

Table 4 Impaired test results per subject group

\begin{tabular}{llll}
\hline $\begin{array}{l}\text { No of } \\
\text { abnormal } \\
\text { tests }\end{array}$ & $\begin{array}{l}\text { Pneumococcal } \\
\text { patients }(\mathbf{n}=79)\end{array}$ & $\begin{array}{l}\text { Meningococcal } \\
\text { patients }(\mathbf{n}=76)\end{array}$ & $\begin{array}{l}\text { Control subjects } \\
(\mathbf{n}=72)\end{array}$ \\
\hline 0 & $24(30)$ & $34(45)$ & $38(54)$ \\
1 & $17(22)$ & $13(17)$ & $22(31)$ \\
2 & $9(11)$ & $8(10)$ & $7(10)$ \\
$\geqslant 3$ & $29(37)$ & $21(28)$ & $5(6)$ \\
\hline \multicolumn{4}{l}{ Values are number (\%) of patients. } \\
\hline
\end{tabular}

Time for the dominant hand $(\mathrm{p}<0.01)$. The frequency of cognitive impairment was higher in the patient group compared with the control group (50 of $155(32 \%)$ vs 4 of $72(5.5 \%)$; $\mathrm{p}<0.001)$. Patients had significantly lower speed scores than controls ( $\mathrm{T}$ scores 46.4 vs 50.0; $\mathrm{p}=0.03$ ). No differences were found between patients and controls on the POMS depression scale ( 5.4 vs $5.7 ; p=0.48$ ). Also, on the questionnaires, patients had worse scores than controls. They had lower scores than controls on the subscales "bodily pain" (69.6 vs 80.0; $p=0.04$ )

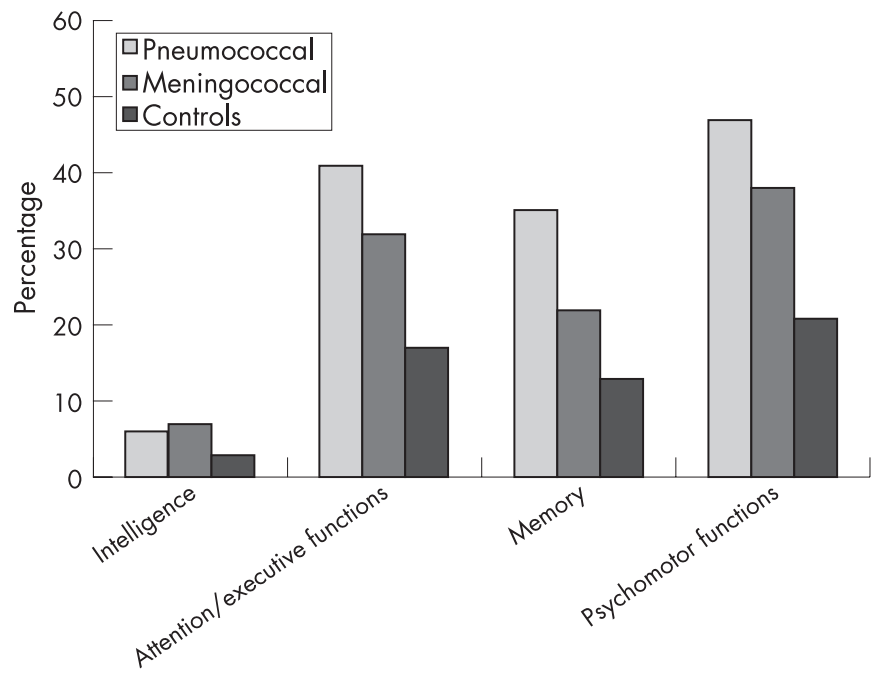

Figure 1 Percentage of subjects with one or more abnormal test results across cognitive domains. 
and "role limitations due to physical health problems" ( 72.7 vs 84.9; $\mathrm{p}<0.01$ ) of the RAND-36.

\section{Causative organism}

Pneumococcal patients were older $(\mathrm{p}<0.001)$ and had a slightly lower level of education compared with meningococcal patients $(\mathrm{p}=0.02)$ although premorbid intelligence did not differ between the patient groups $(\mathrm{p}=0.84)$ (table 2$)$. T scores of neuropsychological tests, corrected for age and education, for both patient groups are shown in table 3 (by definition, mean $\mathrm{T}$ scores in the control group are 50 (SD 10)). MANOVAs within the cognitive domains showed significant group differences between pneumococcal and meningococcal patients for "memory" $(p=0.05)$ but not for "intelligence" $(p=0.90)$, "attention/ executive functions" $(\mathrm{p}=0.24)$ or "psychomotor functions" $(\mathrm{p}=0.24)$. Pneumococcal patients performed worse than meningococcal patients on Wechsler Memory Scale-revised Visual Reproduction percentage retained. Pneumococcal patients tended to have lower speed composite scores than meningococcal patients $(\mathrm{p}=0.08)$.

The numbers of abnormal test results for both patient groups and controls are presented in table 4. Subjects with three or more impaired test results were considered to be "cognitively impaired". A total of 50 patients (32\%) were cognitively impaired. The frequency of cognitive impairment was similar for pneumococcal and meningococcal patients (29 of 79 (37\%) vs 21 of $76(28 \%) ; p=0.24)$.

The frequency of abnormal test results across the various cognitive domains is shown in fig 1 . Pneumococcal patients had significantly more impaired test results in the cognitive domains of attention/executive functions $(p<0.001)$, memory $(p<0.001)$ and psychomotor functions $(\mathrm{p}<0.001)$ compared with controls. Meningococcal patients had more impaired test results than controls on attention/executive functions $(p=0.03)$ and psychomotor functions $(p=0.03)$. Pneumococcal patients tended to have more impaired test results in the cognitive domain of memory than meningococcal patients $(\mathrm{p}=0.06)$.

Pneumococcal patients had higher scores on the depression scale of the POMS compared with meningococcal patients (7.1 vs $5.3 ; p=0.05)$. No significant correlations $\left(p_{s}>0.17\right)$ between POMS depression score and the number of impaired test results or speed score were found in both patient groups, or between the GOS score and number of impaired test scores. Pneumococcal patients had lower scores on the subscale physical functioning of the RAND-36 compared with meningococcal patients $(78.7$ vs $88.1 ; \mathrm{p}<0.01)$. Ratings of physical functioning (RAND-36) and the speed composite score were not significantly correlated $(r=0.15 ; p=0.08)$.

\section{Interval between onset of illness and cognitive testing}

The median interval between onset of illness and cognitive testing was 49.5 months (range 5-164) and did not correlate with performance on the speed composite score $(\mathrm{r}=0.09$; $\mathrm{p}=0.25)$ and number of abnormal test results $(\mathrm{r}=-0.05$; $\mathrm{p}=0.54$ ). Scores on the subscale physical functioning (RAND36) correlated significantly with time since meningitis

\begin{tabular}{lll} 
Table 5 & Impaired test results per treatment group \\
\hline $\begin{array}{l}\text { No of abnormal } \\
\text { tests }\end{array}$ & $\begin{array}{l}\text { Dexamethasone } \\
(\mathbf{n}=46)\end{array}$ & $\begin{array}{l}\text { No dexamethasone } \\
(\mathbf{n}=109)\end{array}$ \\
\hline 0 & $16(35)$ & $42(39)$ \\
1 & $8(17)$ & $22(20)$ \\
2 & $5(11)$ & $12(11)$ \\
$\geqslant 3$ & $17(37)$ & $33(30)$ \\
\hline
\end{tabular}

$(\mathrm{r}=0.30 ; \mathrm{p}<0.001)$. In addition, physical functioning was negatively correlated with number of impaired test results $(\mathrm{r}=-0.20 ; \mathrm{p}=0.02)$.

\section{Adjunctive dexamethasone therapy}

The influence of dexamethasone therapy was recently described in a long term follow-up study of the EDS. ${ }^{6}$ Within the data of the EDS, MANOVAs of neuropsychological domains showed no significant overall group differences between both treatment groups for "intelligence" $(p=0.35)$, "memory" $(p=0.29)$, "attention/executive functioning" $(p=0.11)$ or "psychomotor speed" ( $p=0.71)$; scores on the speed composite score for placebo and dexamethasone treated patients were also similar $(p=0.37)$.

In the current analysis with pooled data, all analyses were performed for patients with and without adjunctive dexamethasone therapy separately; no differences were found in these subanalyses (data not shown). However, MANOVAs showed significant overall differences in the domains "memory" $(p=0.02)$ and "attention/executive function" $(p<0.001)$ but not in "intelligence" $(\mathrm{p}=0.10)$ or "psychomotor speed" $(p=0.41)$. Patients who were treated with steroids had better scores on the Wechsler Memory Scale immediate recall $(\mathrm{T}$ scores 51.8 vs $46.2 ; \mathrm{p}=0.02$ ) and fewer errors on the Wisconsin Card Sorting Test ( $\mathrm{T}$ scores 52.8 vs 47.3; $\mathrm{p}<0.001$ ); however they had lower scores on numerical ability of the GIT ( $\mathrm{T}$ scores 46.4 vs $50.3 ; p=0.04)$. Importantly, there were no significant differences between patients with and without adjunctive dexamethasone therapy within the different causative organisms. The frequency of cognitive impairment for patients with and without adjunctive dexamethasone therapy was similar $(p=0.42)$, as were numbers of abnormal test results (table 5$)$.

\section{Predictors of cognitive impairment}

The nine most important potential predictors of cognitive impairment were subjected to a logistic regression analysis: causative pathogen, sex, years of education, premorbid intelligence, age, Glasgow Coma Scale on admission, presence of cranial nerves palsies at discharge, presence of focal cerebral deficits at discharge and interval between onset of illness and cognitive testing. Male patients after bacterial meningitis were at higher risk for cognitive impairment (odds ratio (OR) 3.08; $95 \%$ CI 1.33 to $7.13 ; \mathrm{p}<0.01$ ). Patients with cranial nerve palsies at discharge were also at higher risk for cognitive impairment (OR 4.73; 95\% CI 1.37 to $16.28 ; \mathrm{p}=0.01$ ). The HosmerLemeshow Goodness of Fit test confirmed the validity of the final multivariate model $\left(\chi^{2}=3.6, \mathrm{df}=8, \mathrm{p}=0.90\right)$.

\section{DISCUSSION}

Our study showed that after bacterial meningitis, patients are at high risk of cognitive impairment. Cognitive impairment was found in one-third of patients, and hence large numbers of patients will continue to have complaints attributable to their illness after the acute phase of the disease.

The prevalence of cognitive impairment in patients after pneumococcal and meningococcal meningitis was similar. This is in contrast with our previous studies in which we described cognitive impairment after pneumococcal meningitis only, ${ }^{4} 10$ but is consistent with a recent retrospective study. ${ }^{9}$ The discrepancy between the present study and our previous reports may be explained by the larger numbers of patients and controls, which has resulted in more statistical power and superior normative reference scores from controls.

We found a diffuse pattern of cognitive impairment, in which cognitive speed played the most important role. Previous studies on cognitive outcome after bacterial meningitis have described impairment of memory, decreased psychomotor performance, impaired attention/executive functions and 
reduction in visuoconstructive capacity. ${ }^{59} \mathrm{~A}$ previous retrospective study involving 59 adults younger than 70 years, 112 years after recovery from bacterial meningitis, found poorer performance in subtests that required plan formation and learning strategies. ${ }^{9}$ The findings of executive deficits in this study may also have been associated with a decline in cognitive speed.

The most important limitation of our study was selection bias. Firstly, patient who could not be reliably assessed with the neuropsychological test battery were excluded (ie, those with severe disability and low scores on the GOS). Low GOS might also reflect severe neuropsychological deficits. Thus severe cases of neuropsychological deficits might not be represented in this study. Secondly, a considerable number of patients were tested in a follow-up study in a randomised, controlled trial, so all of these patients met specific inclusion criteria. However, the baseline characteristics of patients in the EDS were similar to those included in our nationwide cohort of adults with culture proven bacterial meningitis. ${ }^{11}$ Therefore, patients included in the European study are likely to be a representative sample of adults with bacterial meningitis. Thirdly, all patients included in this neuropsychological evaluation underwent lumbar puncture. Negative CSF cultures were found in 11-30\% of patients with bacterial meningitis. ${ }^{12}$ No significant differences in clinical presentation have been reported between patients with culture positive and culture negative bacterial meningitis. ${ }^{1}$ Therefore, it is unlikely that this factor confounded our results.

Patients reported significant improvement in physical impairment in the years after meningitis, while cognitive outcome was not related to time. We found no confounding by higher scores on the depression scale. Although speculative, this may imply that patients adapt to their cognitive impairments, even if these impairments remain stable. The effect of cognitive impairment after bacterial meningitis on longer term outcome ( $>10$ years) and the potential influence on the development of dementia remains to be elucidated.

The use of dexamethasone was not associated with cognitive impairment. This has also been reported by a follow-up study of the EDS. ${ }^{611}$ Results of an experimental meningitis model raised concerns about a possible harmful effect of adjunctive dexamethasone therapy on cognition in patients after bacterial meningitis. ${ }^{23}$ This study in infant rats showed that adjunctive dexamethasone aggravated neuronal apoptosis in the hippocampal dentate compared with antibiotic therapy alone. ${ }^{22}$ Since adjunctive dexamethasone therapy should be routine in adults with bacterial meningitis, this is important information. ${ }^{261123}$

In summary, our results showed that approximately onethird of adult survivors of bacterial meningitis experience subtle cognitive impairment which consists mainly of slight mental slowness. Male sex and cranial nerve palsy at discharge were risk factors for cognitive impairment. Over the years, patients tended to report fewer complaints, but the cognitive impairment, if present, does not seem to improve once the subacute phase has elapsed.

\section{ACKNOWLEDGEMENTS}

We are indebted to many physicians in the Netherlands for their cooperation.

\section{Authors' affiliations}

Martine Hoogman, Diederik van de Beek, Martijn Weisfelt, Jan de Gans,

Ben Schmand, Department of Neurology, Centre of Infection and Immunity Amsterdam (CINIMA), Academic Medical Centre, Amsterdam, the

Netherlands

Funding: This study was supported by a grant from the Meningitis Research Foundation, UK (DvdB grant No 03/03). The Dutch Meningitis Cohort was supported in part by an unrestricted research grant from Roche Pharmaceuticals (JdG). The European Dexamethasone Study was supported in part by a research grant from Organon NV (JdG). DvdB is supported by personal grants from the Netherlands Organisation for Health Research and Development (ZonMw) (NWO-Veni grant 2006 (916.76.023), NWO-Rubicon grant 2006 (019.2006.1.310.001)) and the Dr Jan Meerwaldt Foundation. MH, MW and BS have received no specific funding for this study.

Competing interests: None.

\section{REFERENCES}

1 van de Beek D, de Gans J, Spanjaard L, et al. Clinical features and prognostic factors in adults with bacterial meningitis. N Engl J Med 2004;351:1849-59.

2 van de Beek D, de Gans J, Tunkel AR, et al. Community-acquired bacterial meningitis in adults. N Engl J Med 2006;354:44-53.

3 Weisfelt $M$, van de Beek D, Spanjaard L, et al. Clinical features, complications, and outcome in adults with pneumococcal meningitis: a prospective case series. Lancet Neurol 2006;5:123-9.

4 van de Beek D, Schmand B, de Gans J, et al. Cognitive impairment in adults with good recovery after bacterial meningitis. J Infect Dis 2002;186:1047-52.

5 Merkelbach S, Sittinger H, Schweizer I, et al. Cognitive outcome after bacterial meningitis. Acta Neurol Scand 2000;102:118-23.

6 Weisfelt $M$, Hoogman $M$, van de Beek D, et al. Dexamethasone and long-term outcome in adults with bacterial meningitis. Ann Neurol 2006;60:456-68.

7 Bohr V, Rasmussen N, Hansen B, et al. Pneumococcal meningitis: an evaluation of prognostic factors in 164 cases based on mortality and on a study of lasting sequelae. J Infect 1985;10:143-57.

8 Naess A, Halstensen A, Nyland $\mathrm{H}$, et al. Sequelae one year after meningococcal disease. Acta Neurol Scand 1994;89:139-42.

9 Schmidt H, Heimann B, Djukic M, et al. Neuropsychological sequelae of bacterial and viral meningitis. Brain 2006;129:333-45.

10 Weisfelt $M$, van de Beek D, Hoogman $M$, et al. Cognitive outcome in adults with moderate disability after pneumococcal meningitis. J Infect 2006;52:433-9.

11 de Gans J, van de Beek D. Dexamethasone in adults with bacterial meningitis. N Engl J Med 2002;347:1549-56.

12 Rey A. L'examen clinique en psychology. Paris: Presses Universitaires de France, 1964.

13 Wilson B, Cockburn J, Baddeley A. Rivermead behavioral memory test. Reading, UK: Thames Valley Test Company, 1985.

14 Stroop J. Studies of interference in serial verbal reactions. J Exp Psychol 1935; 18:643-62.

15 Reitan R. Trail making test. Manual for administration and scoring. Tuscon: Reitan Neuropsychological Laboratory, 1992.

16 Alpherts WCJ, Aldenkamp AP. Fepsy. Heemstede: Instituut voor epilepsiebestrijding, 1994.

17 Lutelijn F, van der Ploeg F. Groningen Intelligentie Test. Lisse: Swets and Zeitlinger, 1983.

18 Schmand B, Lindeboom J, van Harskamp F. De Nederlandse Leestest voor volwassenen. Lisse: Swets and Zeitlinger, 1992.

19 Lezak MD, Howieson BD, Loring DW. Neuropsychological assessment, 4th edn. New York: Oxford University Press, 2004.

20 Brazier JE, Harper R, Jones NMB, et al. Validating the Sf-36 Health Survey Questionnaire-new outcome measure for primary care. BMJ 1992;305:160-4.

21 van der Zee K, Sanderman R. Het meten van de algemene gezondheidstoestand met de RAND-36. Groningen: Groningen University, 1993.

22 Leib SL, Heimgartner C, Bifrare YD, et al. Dexamethasone aggravates hippocampal apoptosis and learning deficiency in pneumococcal meningitis in infant rats. Pediatr Res 2003;54:353-7.

23 van de Beek D, de Gans J. Dexamethasone in adults with community-acquired bacterial meningitis. Drugs 2006;66:415-27. 